\title{
Synergistic effect of immune checkpoint blockade and anti-angiogenesis in cancer treatment
}

\author{
Ming Yi ${ }^{1}$, Dechao Jiao ${ }^{2}$, Shuang Qin ${ }^{1}$, Qian $\mathrm{Chu}^{1}$, Kongming $\mathrm{Wu}^{1,2^{*}} \mathrm{D}$ and Anping $\mathrm{Li}^{2^{*}}$
}

\begin{abstract}
Immune checkpoint inhibitor (ICl) activates host's anti-tumor immune response by blocking negative regulatory immune signals. A series of clinical trials showed that $\mathrm{ICl}$ could effectively induce tumor regression in a subset of advanced cancer patients. In clinical practice, a main concerning for choosing $\mathrm{ICl}$ is the low response rate. Even though multiple predictive biomarkers such as PD-L1 expression, mismatch-repair deficiency, and status of tumor infiltrating lymphocytes have been adopted for patient selection, frequent resistance to $\mathrm{ICl}$ monotherapy has not been completely resolved. However, some recent studies indicated that $\mathrm{ICI}$ resistance could be alleviated by combination therapy with anti-angiogenesis treatment. Actually, anti-angiogenesis therapy not only prunes blood vessel which is essential to cancer growth and metastasis, but also reprograms the tumor immune microenvironment. Preclinical studies demonstrated that the efficacy of combination therapy of $\mathrm{ICl}$ and anti-angiogenesis was superior to monotherapy. In mice model, combination therapy could effectively increase the ratio of anti-tumor/pro-tumor immune cell and decrease the expression of multiple immune checkpoints more than PD-1. Based on exciting results from preclinical studies, many clinical trials were deployed to investigate the synergistic effect of the combination therapy and acquired promising outcome. This review summarized the latest understanding of $\mathrm{ICl}$ combined antiangiogenesis therapy and highlighted the advances of relevant clinical trials.
\end{abstract}

Keywords: Immune checkpoint inhibitor, PD-1, PD-L1, CTLA-4, VEGF, Anti-angiogenesis, TKI, Tumor immune microenvironment

\section{Background}

Immune checkpoint molecules mainly includes programmed cell death protein 1 (PD-1) and cytotoxic T-lymp hocyte antigen-4 (CTLA-4) [1-4]. As the vital components of immune homeostasis, immune checkpoint molecules downregulate magnitude of immune response and participate in peripheral tolerance [5]. However, upregulated immune checkpoint signaling pathways such as PD-1/PD-L1 protect cancer cell from immune surveillance [6]. Therefore, immune checkpoint molecules and their ligands are ideal anti-cancer treatment targets. It is well established that anti-PD-1/PD-L1 upregulates Ras-Raf-MEK-ERK and PI3KAKT signaling pathways in immune cells by blocking PD-1/

\footnotetext{
* Correspondence: kmwu@tjh.tjmu.edu.cn; li_anping@yahoo.com ${ }^{1}$ Department of Oncology, Tongji Hospital of Tongji Medical College, Huazhong University of Science and Technology, Wuhan 430030, China ${ }^{2}$ Department of Interventional Radiology, The First Affiliated Hospital of Zhengzhou University, Zhengzhou 450052, China
}

PD-L1 axis [7]. As a result, anti-PD-1/PD-L1 therapy restores $\mathrm{T}$ cell from exhausted status and enhances tumorkilling activity [8]. Relatively, mechanisms by which antiCTLA-4 therapy destroys cancer cell are still controversial. It is generally believed that anti-CTLA-4 recovers the co-stimulatory signaling pathway CD28-B7 which is usually hijacked by CTLA-4 in tumor microenvironment $[9,10]$. Additionally, it is proposed that anti-CTLA-4 could directly eliminate regulatory $\mathrm{T}$ (Treg) cell by antibody-dependent cell-mediated cytotoxicity [11-13].

Compared with immune checkpoint inhibitor (ICI), anti-angiogenesis therapy attracted intensive attention earlier. Angiogenesis, mainly indicating the generation of new vessels from pre-existing ones, occurs in many physiological processes (e.g. wound healing) [14]. In the meanwhile, angiogenesis participates in the growth and metastasis of solid tumor [15]. Due to the characteristics of the rapid division and growth, tumor cell consumes a

(c) The Author(s). 2019 Open Access This article is distributed under the terms of the Creative Commons Attribution 4.0 International License (http://creativecommons.org/licenses/by/4.0/), which permits unrestricted use, distribution, and 
large amount of oxygen and nutrients. Besides, active metabolism with disproportional blood supply leads to hypoxia and acidosis in tumor bed [15, 16]. Subsequently, hypoxia induces tumor and stroma cells to secret multiple pro-angiogenic factors such as vascular endothelial growth factor (VEGF), basic fibroblast growth factor (bFGF), and matrix metalloproteinase (MMP) [17]. As a result, the local balance of pro-angiogenic factors and anti-angiogenic factors is disturbed and multiple angiogenic pathways are activated [18]. However, due to the persistent hypersecretion of pro-angiogenic factors in tumor microenvironment, vessel maturation process is impeded [19]. Abnormal angiogenesis leads to the lack of pericyte coverage and leaky nascent vessels [20,21]. Disorganized and leaky vessels result in increased vascular permeability and interstitial fluid pressure [22].

The initial aim of anti-angiogenesis therapy is to reduce blood supply and starve tumor cell of oxygen and nutrients [23]. However, no significant improvements in outcomes were observed in patients undergoing antiangiogenesis therapy alone. Vessel normalization theory provides a novel perspective in anti-angiogenesis and indicates potential synergistic effect in combination with other therapies. This review focused on the application of ICI combined with anti-angiogenesis therapy.

\section{The influence of angiogenesis on ICI therapy}

\section{The status of tumor infiltrating lymphocytes determines} the efficacy of ICI

Tumor infiltrating lymphocyte (TIL) is one of the most important components for tumor-killing activity. For ICI therapy especially anti-PD-1/PD-L1 intervention, pre-existing TIL is the necessary precondition for potent tumor regression. Based on the status of pre-existing TIL, tumor microenvironments are classified into three types: (I) immune inflamed type, where dense functional $\mathrm{CD}^{+} \mathrm{T}$ cells infiltrate; (II) excluded infiltration type, where abnormal angiogenesis and immunosuppressive reactive stroma prevent the infiltration of T cell; (III) immune ignorance type, where tumor mutation burden and the expression of antigen presentation machinery marker are low [24]. It was verified that the tumors belonging to immune inflamed type were more sensitive to ICI therapy than two other types [25]. Moreover, treatment enhancing $\mathrm{T}$ cell infiltration could promote the effect of ICI $[26,27]$.

\section{Angiogenesis affects the status of TIL}

In cancer-immunity cycle, the presentation of neoantigen determines the generation of tumor specific $\mathrm{T}$ cell clones. Then, $\mathrm{T}$ cells with specific $\mathrm{T}$ cell receptor (TCR) traffic to and infiltrate into tumor. TIL recognizes neoantigen and kills tumor cell in immunosupportive tumor microenvironment $[28,29]$. For most growing solid tumors, hyperactive angiogenesis contributes to immunosuppressive microenvironment by affecting multiple immune steps (Fig. 1) [30, 31].

On the one hand, abnormal angiogenesis decreases the abundance and function of anti-tumor lymphocytes. Firstly, leaky nascent vessels and loose pericyte coverage result in high interstitial fluid pressure which means greater pressure difference to overcome for $\mathrm{T}$ cell infiltration. Rare $\mathrm{T}$ cell could cross physical barrier and infiltrates into tumor bed [32]. Secondly, neo-vasculatures tend to lack some adhesion molecules for example vasculature cell adhesion molecule-1 (VCAM-1). Downregulated adhesion molecules further impair the extravasation of $\mathrm{T}$ cell [32]. Thirdly, neo-vasculatures could not compensate for increased oxygen consumption and concurrent hypoxia directly undermine the functions of TIL. Hypoxia upregulates some inhibitory signals for anti-tumor immune response such as PD-L1, indoleamine 2, 3-dioxygenase (IDO), interleukin-6 (IL-6), and interleukin-10 (IL-10) [14, 33]. In addition, circulating VEGF impedes the maturation and function of dendritic cell (DC) to help tumor escape immune surveillance $[34,35]$.

On the other hand, hyperactive angiogenesis increases the abundance of pro-tumor lymphocytes. As the consequence of abnormal tumor vessel, tumor hypoxia induces upregulation of chemokine (C-C motif) ligand-22 and chemokine (C-C motif) ligand-28, which recruit Treg into tumor [36, 37]. Besides, hypoxic tumor microenvironment promotes the polarization of tumor-associated macrophage (TAM) to M2-like phenotype [38]. Thirdly, the expression of Fas ligand (FasL) on tumor endothelial barrier selectively eliminates effector $\mathrm{CD}^{+} \mathrm{T}$ cells rather than Treg, due to the high expression of cellular FLICE-inhibitory protein (c-FLIP) expression on Treg [39]. In summary, angiogenesis participates in tumor growth and immune evasion by multiple manners.

\section{Anti-angiogenesis agents: The natural ally of $\mathrm{ICI}$ Main anti-angiogenesis agents}

Solid tumors tend to secret multiple pro-angiogenetic factors such as VEGF (also known as VEGF-A), hepatocyte growth factor, and platelet derived growth factor. Among these factors, VEGF plays a core role in angiogenesis [21, 40]. The angiogenetic signal of VEGF is mainly transducted by its receptor VEGFR2 [41, 42]. VEGFR2 contains a ligand-binding domain with 7 immunoglobulin-like structures, a trans-membrane domain, and a tyrosine kinase domain [43]. On the one hand, VEGF-VEGFR2 promotes secretion of von Willebrand factor (vWF), proliferation and migration of endothelial cell (EC) by activating downstream PLC $\gamma$-PKC-Raf-MAPK and Grb2Gab1-MAPK/PI3K-Akt signaling pathways [44]. On the other hand, VEGF-VEGFR2 could increase vascular permeability by activating VEGFR2-TSAd-Src-cadherin and 


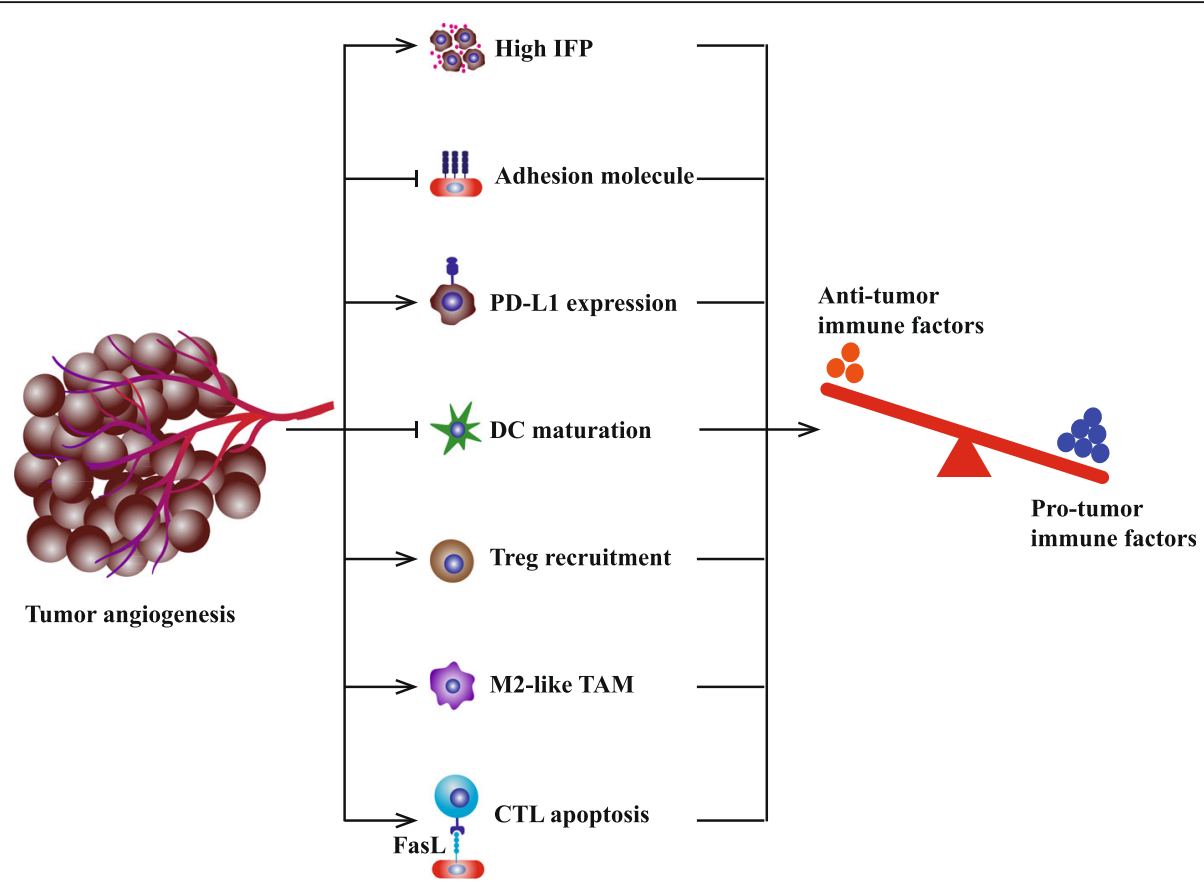

Fig. 1 Tumor angiogenesis induces the formation of immunosuppressive tumor microenvironment. Firstly, leaky nascent vessels and loose pericyte coverage result in high interstitial fluid pressure (IFP) which means greater pressure difference to overcome for T cell infiltration. Secondly, neo-vasculatures tend to lack some adhesion molecules for example vasculature cell adhesion molecule-1 (VCAM-1). Thirdly, hypoxia upregulates some inhibitory signals for anti-tumor immune response such as PD-L1, indoleamine 2, 3-dioxygenase (IDO), interleukin-6 (IL-6), and interleukin-10 (IL-10). In addition, circulating VEGF impedes the maturation and function of dendritic cell (DC). Besides, tumor hypoxia induces upregulation of chemokine (C-C motif) ligand-22 and chemokine (C-C motif) ligand-28, which recruit Treg into tumor [36, 37]. Moreover, hypoxic tumor microenvironment promotes the polarization of tumor-associated macrophage (TAM) to M2-like phenotype. Lastly, the expression of Fas ligand (FasL) on tumor endothelial barrier selectively eliminates effector $C D 8^{+} \mathrm{T}$ cells rather than Treg, due to the high expression of cellular FLICE-inhibitory protein (c-FLIP) expression on Treg. In summary, angiogenesis render accumulating pro-tumor immune cells and decreasing antitumor immune cells, inducing the formation of immunosuppressive tumor microenvironment

PI3K-Akt-eNOS-NO signaling pathways (Fig. 2a) [23, 44]. Therefore, VEGF and its receptor VEGFR2 are predominant targets for the development of anti-angiogenesis agents. Anti-VEGF monoclonal antibody (mAb) bevacizumab is the first anti-angiogenesis agent which is approved for multiple cancers including metastatic colorectal cancer, metastatic non-squamous non-small cell lung cancer, metastatic renal cell carcinoma, recurrent glioblastoma, recurrent ovarian cancer, recurrent/metastatic cervical cancer [45]. Following the invention of bevacizumab, a variety of VEGFVEGFR targeted agents come out. Apart from anti-VEGF $\mathrm{mAb}$, there are other three approaches to inhibit VEGFVEGFR signaling pathway: (I) decoy VEGF-trap receptor such as aflibercept [46]; (II) anti-VEGFR2 mAb such as ramucirumab [47]; (III) tyrosine kinase inhibitor (TKI) which interferes intracellular signal transduction of VEGF such as axitinib, sorafenib, sunitinib, and vatalanib [48-51]. Moreover, based on chimeric antigen receptor (CAR) T cell technology, Chinnasamy et al. developed anti-VEGFR2 CAR $\mathrm{T}$ cell to retard tumor growth [52]. Anti-VEGFR2 CAR-T therapy is verified as an effective strategy inducing tumor regression but its effect needs further investigation in human.

\section{Anti-angiogenesis: From tumor starvation to vessel normalization}

For most species, the formation of functional vessel needs maturation process [19]. In the absence of VEGF, nascent vessels undergo a series of modification procedures including basement membrane deposition, EC-EC junction formation, and pericyte coverage [19]. Driven by the persistent hypersecretion of VEGF, tumor vessels do not possess tight EC-EC conjunction, sufficient pericyte coverage, and lack intact basement membrane $[53,54]$. The survival of these vessel is highly dependent on activated VEGF-VEGFR2 signaling pathway [55].

Originally, anti-angiogenesis agents were developed to interfere neo-vascularization and starve tumor, but they did not yield satisfactory effect [53]. Presumably under selective pressure, tumor with excessively pruned blood vessel are prone to transform to the phenotype tolerable to hypoxia, rendering increased invasiveness and metastasis ability $[56,57]$. In spite of the unsatisfactory efficacy of monotherapy, it was found anti-angiogenesis could be used as a sensitizer in combination with other therapies $[58,59]$. However, there is a paradox that the elimination 

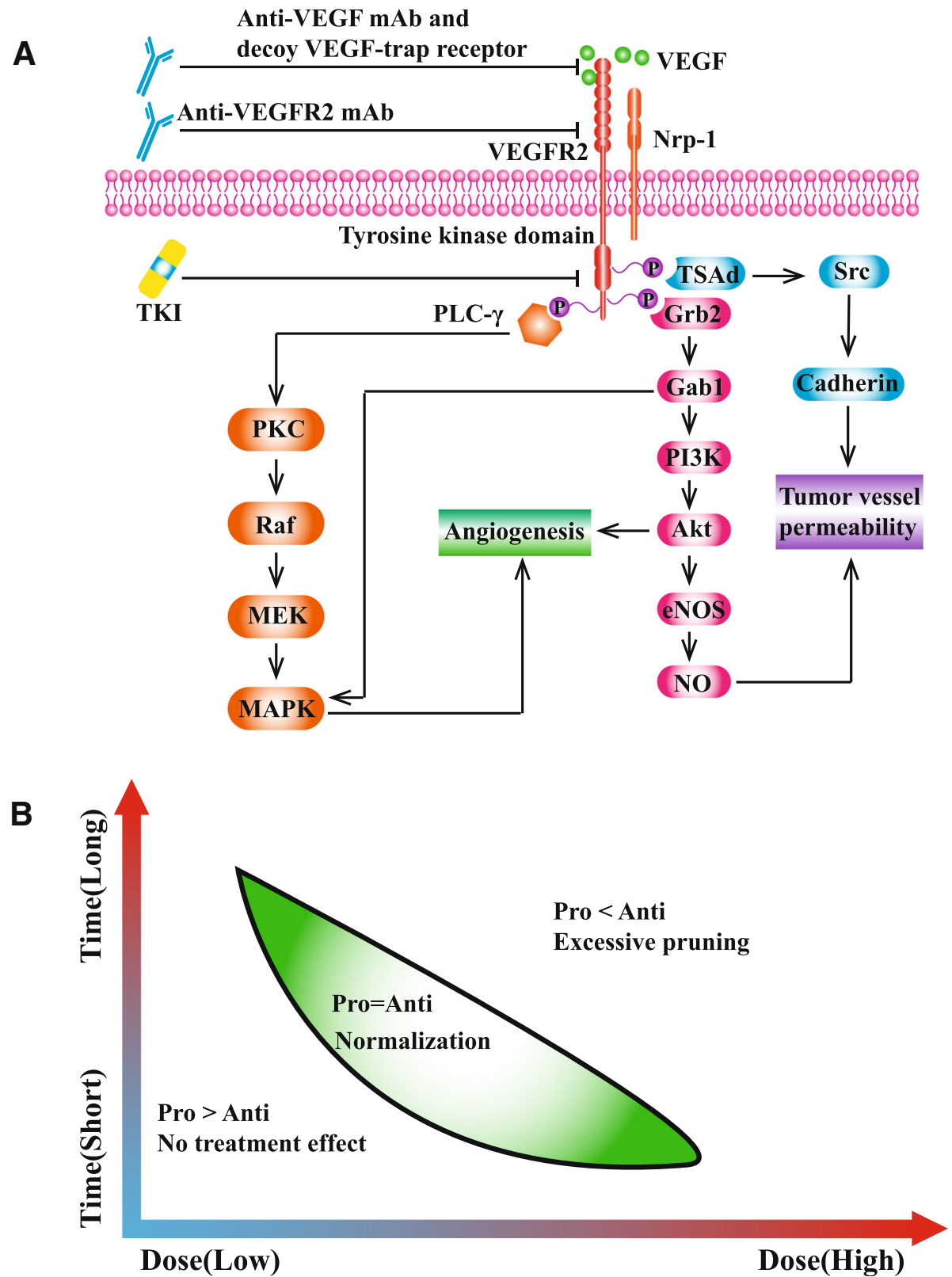

Fig. 2 a Main angiogenesis pathways and anti-angiogenesis agents. VEGF-VEGFR2 promotes the proliferation and migration of endothelial cell primarily by activating downstream PLCY-PKC-Raf-MAPK and Grb2-Gab1-MAPK/PI3K-Akt signaling pathways. In addition, VEGF-VEGFR2 could increase vascular permeability by activating VEGFR2-TSAd-Src-cadherin and PI3K-Akt-eNOS-NO signaling pathways. Anti-angiogenesis agents consist of three types: (I) anti-VEGF monoclonal antibody (mAb) such as bevacizumab and decoy VEGF-trap receptor such as aflibercept; (II) anti-VEGFR2 mAb (ramucirumab); (III) VEGFR tyrosine kinase inhibitor (TKI). b Normalization window of anti-angiogenesis treatment. When pro-angiogenic (pro) factors balance with antiangiogenic (anti) factors, abnormal tumor vessels transform into normal-like phenotype (green). Vessel normalization is a transient status changing along with the time and dose of treatment

of tumor vessel simultaneously restrains the delivery of drug and oxygen [53]. Jain established a model to describe the transient status of tumor vessel undergoing anti-angiogenesis: vessel normalization [53]. In the model, when pro-angiogenic factors balance with antiangiogenic factors, abnormal tumor vessels transform into a normal-like phenotype with characteristics including increased perfusion, pericyte coverage, and decreased hypoxia $[53,60,61]$. Notably, vessel normalization status depends on the schedule and dose of treatment (Fig. 2b). Huang et al. conducted a study to investigate the relationship between anti-angiogenesis dose and efficacy. The results demonstrated that lower dose of anti-angiogenesis agent was superior to higher 
dose treatment in inducing homogeneous tumor vessel normalization [62]. We proposed that higher dose anti-angiogenesis might result in rapider vessel pruning and shorter normalization window.

\section{Anti-angiogenesis: Reprograming tumor immune microenvironment}

A growing body of evidence demonstrated that appropriate anti-angiogenesis administration could convert tumor immune environment from immunosuppressive to immunosupportive status [63, 64]. Normalized tumor vascular network could directly alleviate hypoxia and promote $\mathrm{T}$ cell infiltration. Alleviated hypoxia preferentially induces polarization of TAM to M1-like phenotype [62]. Besides, vessel normalization decreases the recruitment of Treg and myeloid-derived suppressor cell (MDSC) [14, 65]. In addition, anti-VEGF agents block the inhibitory signal for DC differentiation and decrease overall MDSC pool [66]. Lastly, hypoxia-induced inhibitory immune signals such as PD-L1 could be downregulated by improved perfusion [67].

\section{ICI plus anti-angiogenesis therapy in preclinical studies}

Tumor immune escape closely relates to angiogenesis. In turn, tumor angiogenesis highly depends on immunosuppressive microenvironment. Activated $\mathrm{T}$ cell secrets interferon- $\gamma$ (IFN- $\gamma$ ) which could directly promote tumor vessel normalization and regression by IFN- $\gamma$ receptor on tumor endothelial cell (Fig. 3) [68-70]. Based on the interaction between tumor immunity and angiogenesis, it is speculated that anti-angiogenesis might enhance the efficacy of ICI. As early as 2013, Yasuda et al. observed the synergistic effect between ICI and anti-angiogenesis in mice bearing colon adenocarcinoma [71]. Subsequently, $\mathrm{Wu}$ et al. verified that ICI plus anti-angiogenesis could effectively prolong overall survival (OS) in mice bearing kidney and mammary tumors [72]. However, apart from decreased interstitial fluid pressure and correspondingly improved $\mathrm{T}$ cell infiltration, we could not rule out other mechanisms by which ICI and anti-angiogenesis synergistically kill tumor cell. Thus, further explorations should be conducted in expanding models. To date, multiple mechanisms have been found to relate to synergistic effect.

\section{Blocking VEGF-induced immune checkpoint expression}

Meder et al. conducted a preclinical study by genetically engineered small-cell lung cancer (SCLC) mouse [73]. All SCLC-bearing mice were randomly assigned into five groups and received the following therapies: (I) phosphate buffered saline (vehicle); (II) IgG; (III) anti-VEGF mAb (B20-4.1.1-PHAGE); (IV) anti-PD-L1 mAb (clone 6E11); (V) anti-VEGF plus anti-PD-L1 [73]. Among 5 groups, combination therapy group possessed the best survival data [73]. Moreover, compared with mice sensitive to anti-PD-L1, the abundance of exhausted $\mathrm{T}$ cell $\left(\mathrm{PD}-1^{+} / \mathrm{TIM}^{-} 3^{+} / \mathrm{LAG}-3^{+} \mathrm{T}\right.$ cell) significantly increased in mice resistant to anti-PD-L1 [73]. However, the increased ratio of exhausted $\mathrm{T}$ cells could be reversed by following anti-VEGF plus anti-PD-L1 treatment [73]. To confirm the influence of VEGF on immune checkpoint expression, human $\mathrm{T}$ cell was obtained from peripheral blood of SCLC patients [73]. After stimulation with VEGF, the expression of PD-1 and TIM-3 on T cell was significantly upregulated [73].

In line with the finding of Meder and colleagues, Voron et al. observed that anti-VEGF could selectively inhibit the expression of immune checkpoint molecules (e.g. PD-1, CTLA-4, and TIM-3) on intratumoral $\mathrm{CD}^{+}{ }^{+} \mathrm{T}$ cell [74]. Voron et al. found that VEGF could upregulate the expression of PD-1 by activating VEGFR2-PLC $\gamma$-calcineurinNFAT signaling pathway [74]. Therefore, anti-PD-1 therapy together with anti-VEGF could effectively block PD-1/ PD-L1 axis and synergistically suppress tumor growth, especially for tumor with VEGF hypersecretion [74].

\section{Impairing IFN- $\gamma$-mediated negative feedback}

Apart from VEGF signaling pathway, angiopoietin-2 (ANGPT2)/Tie 2 is another pro-angiogenic pathway which relates with resistance to anti-VEGF treatment [7577]. Schmittnaegel et al. confirmed that the dual blockade of VEGF and ANGPT2 by bispecific antibody A2V provided a more potent therapeutic effect than monotherapy [78]. In the meanwhile, the treatment effect of dual blockade could be further enhanced by anti-PD-1 treatment [78]. In this preclinical study, multiple tumor bearing mouse models were employed including transgenic or transplanted breast cancer, pancreatic neuroendocrine cancer, melanoma, and colorectal adenocarcinoma models [78]. After A2V treatment, the abundance of anti-tumor immune cells including mature DC, M1-like phenotype TAM, IFN $-\gamma^{+} / \mathrm{CD} 9^{+} \mathrm{CD} 8^{+} \mathrm{T}$ cell increased [78]. In the meanwhile, increased perivascular $\mathrm{CD}^{+} \mathrm{T}$ cells accompanied the high expression of PD-L1 on tumor cell because of IFN- $\gamma$-mediated negative feedback regulatory mechanism [78]. Combination therapy of anti-PD-1 and A2V blocked the negative feedback loop and magnified the immune response [78]. The results showed that more than $30 \%$ mice receiving combination therapy possessed prolonged OS compared with A2V therapy [78].

\section{Inducing high endothelial venule formation}

Allen et al. investigated the efficacy of combination therapy of anti-PD-L1 (anti-PD-L1 mAb: B20S) and anti-VEGFR2 (anti-VEGFR2 mAb: DC101) in mice bearing pancreatic neuroendocrine tumor, mammary carcinoma, and glioblastoma [79]. Combination therapy showed a great advantage in tumor control and OS over monotherapy in pancreatic 


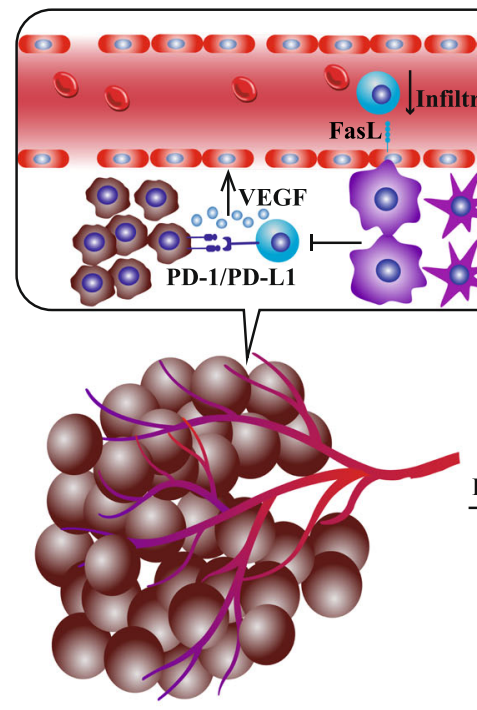

Pre-treatmemt: angiogenesis

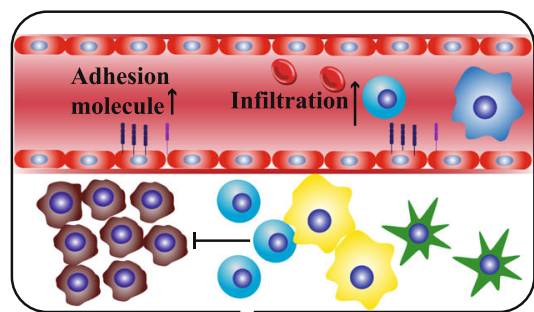

ICI plus anti-angiogenesis

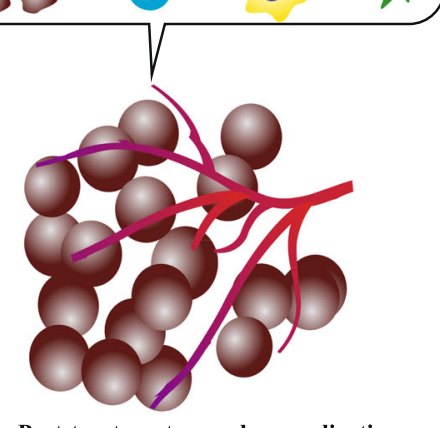

Post-treatment: vessel normalization
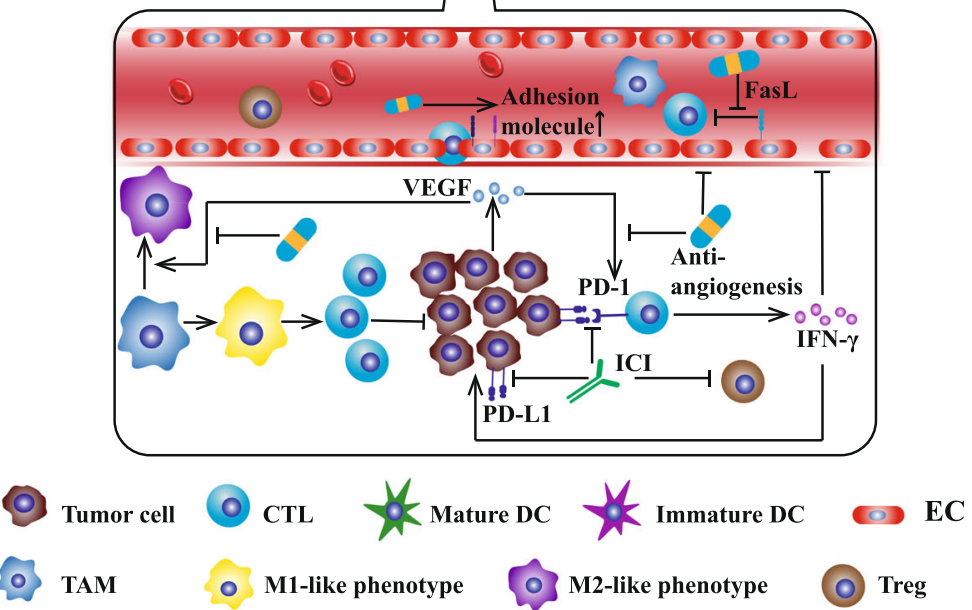

Fig. 3 Mutual regulation of tumor vessel normalization and immune microenvironment reprogramming. Tumor angiogenesis leads to an immunosuppressive microenvironment by decreasing the ratio of anti-tumor/pro-tumor immune cell and undermining the function of cytotoxic T lymphocyte (CTL). Anti-angiogenesis induces tumor vessel normalization and improves blood perfusion. Alleviated hypoxia decreases PD-L1 expression on tumor cell while blocked VEGF signal downregulates immune checkpoint expression (e.g. PD-1) on CTL. In the meanwhile, activated immune response-derived inflammatory factors such as interferon- $\gamma$ (IFN- $\gamma$ ) promotes vessel normalization and regression. Interaction between vessel normalization and immune microenvironment reprogramming could be regulated by anti-angiogenesis agents (bevacizumab or VEGFR-TKI such as axitinib, sorafenib, sunitinib, and vatalanib) and ICI (especially anti-PD-1/PD-L1 mAb). After combination therapy, immunosuppressive microenvironment is transformed to immunosupportive microenvironment which possesses increased CTL, M1-like phonotype macrophage, adhesion molecule, mature dendritic cell (DC), and decreased regulatory T cell (Treg). Abbreviations: TAM, tumor associated macrophage; EC, endothelial cell

neuroendocrine tumor and mammary carcinoma but for glioblastoma [79]. After 2 weeks treatment of anti-PD-L1 plus anti-VEGFR2, the level of IFN- $\gamma^{+} \mathrm{CD}^{+}$and IFN- $\gamma^{+}$ $\mathrm{CD}^{+} \mathrm{T}$ cell increased by twofold in pancreatic neuroendocrine tumor and mammary carcinoma. However, IFN $-\gamma^{+} \mathrm{CD}^{+} \mathrm{T}$ cell modestly increased in just $50 \%$ of glioblastomas [79]. As the direct barrier for $\mathrm{T}$ cell extravasation, intratumoral vessel was speculated as the primary factor contributing to the impeded $\mathrm{T}$ cell infiltration in glioblastomas [79]. Apart from more intact pericyte coverage, vessel in pancreatic neuroendocrine tumor and mammary carcinoma was thickened with plump endothelial cells rather than flat endothelial cells, displaying the unique characteristic of high endothelial venule (HEV) [79]. Immunohistochemical analysis confirmed this phenotype transformation of endothelial cell. It is generally believed that HEV is associated with lymphocyte homing [80-82]. Similarly, it was speculated that intratumoral HEV promoted T cell infiltration into tumor [83]. LTßR signaling pathway is essential to sustain HEV phenotype [79]. Activating LT $\beta R$ signaling pathway by its agonist during combination therapy could effectively 
eliminate glioblastoma, indicating the vital role of $\mathrm{HEV}$ formation in combination therapy [79].

\section{ICI plus anti-angiogenesis therapy in clinical studies}

As discussed above, the interaction between immunity and angiogenesis renders tumor immune escape and treatment resistance. Based on the encouraging results of preclinical studies, many clinical studies have been conducted to investigate the synergistic effect of ICI plus anti-angiogenesis therapy in patients (Table 1). Schmidt et al. established a mathematical model to evaluate synergistic effect of multiple anti-PD-1-based combination therapies including anti-PD-1 plus chemotherapy, anti-angiogenesis, or antiCTLA-4 treatment [84]. By subtracting the independent contributions of combination therapies from overall treatment effect, it was calculated that anti-PD-1 plus antiangiogenesis therapy possessed the strongest synergistic effect among all combination strategies [84].

\section{Anti-CTLA-4 combined with anti-VEGF mAb}

NCT00790010 is a phase I clinical trial to explore the effect of ipilimumab (anti-CTLA-4) plus bevacizumab (anti-VEGF) in metastatic melanoma patients [85]. All 46 recruited patients were classified into 4 cohorts and received different dosages of combination therapy [85]. It was observed that combination therapy significantly promoted upregulation of CD31, E-selectin, VCAM-1, and other adhesion molecules on intratumoral endothelia cell $[85,86]$. In the same time, trafficking of cytotoxic T cell and mature DC were enhanced [85]. Compared with the results of previous studies, patients undergoing combination therapy showed a great advantage in prognosis (median OS, ipilimumab plus bevacizumab vs. ipilimumab: 25.1 vs. 10.1 months) $[85,87]$. Further exploration revealed that the favorable effect of combination therapy might derive from induced immune response to galectin-1 (Gal-1) [88]. Gal-1 is a versatile molecule participating in proliferation, invasion, immune escape, and angiogenesis processes $[89,90]$. Patients' plasma samples were collected to detect the titer of anti-Gal-1 antibody. The results showed that $62.5 \%$ of complete response/partial response patients had increased anti-Gal-1 antibody titer ( $\geq 1.5$ fold), while just $36.4 \%$ of stable disease patients and $23.1 \%$ of progressive disease patients had increase in anti-Gal-1 antibody titer after treatment [89]. Different responses to combination therapy were attributed to distinct anti-Gal-1 immune responses [88]. It was proposed that two factors leaded to the emergency of anti-Gal-1 antibody. On the one hand, anti-VEGF could upregulate the generation of Gal-1 [91]. On the other hand, anti-CTLA-4 increases the phenotypes of $\mathrm{T}$ cell clones. The two factors elevate the probability of Gal-1 recognition by antigen presentation cell [88]. In addition, two other clinical trials (NCT02210117 and
NCT01950390) investigating the effect of combination therapy of ipilimumab plus bevacizumab are ongoing. These two clinical trials involved metastatic kidney cancer and stage III-IV melanoma patient respectively.

\section{Anti-PD-L1 combined with anti-VEGF mAb}

Inspired by the significantly synergistic effect of anti-CTLA-4 plus anti-VEGF therapy, Wallin et al. conducted the clinical study (NCT 01633970) to explore the efficacy of anti-PD-L1 combined with anti-VEGF [26]. NCT01633970 is a phase $1 \mathrm{~b}$ study aiming to investigate the safety and pharmacology of atezolizumab plus bevacizumab or chemotherapy [26]. 10 metastatic renal cell cancer patients received 1 cycle bevacizumab monotherapy followed by combination therapy until disease progression or unacceptable adverse event [26]. 8 of 10 patients showed partial response or stable disease [26]. The results of this small cohort were significantly better than previous monotherapy studies [92, 93]. Compared with tumor samples from patients at baseline or post bevacizumab monotherapy, the expression of CD8, PD-L1, and major histocompatibility complex-I (MHC-I) markedly increased after combination therapy [26]. The transformation to hot tumor was associated with increased expression of CX3CL1 which participated in the recruitment of peripheral $\mathrm{CD}^{+} \mathrm{T}$ cells [26]. Dynamic TCR sequencing analysis demonstrated evolving TCR repertoire during treatment [26]. The emergency of new clones relates to trafficking of tumor specific $\mathrm{T}$ cell and contributes to tumor control [26].

In 2018, the results of the phase 3 study IMpower150 (NCT02366143) were reported. This study was aimed to evaluate the effect of combination therapy consisting of atezolizumab, bevacizumab, and chemotherapy in treatment-naïve metastatic non-squamous non-smallcell lung cancer patients [94]. Among total 2166 enrolled patients, 400 patients received atezolizumab plus bevacizumab plus carboplatin plus paclitaxel therapy (ABCP group) while other 400 patients received bevacizumab plus carboplatin plus paclitaxel therapy (BCP group) [94]. Objective response rate (ORR) of $A B C P$ group was significantly higher than BCP group (ORR: $63.5 \%$ vs. 48.0, $95 \%$ CI: $58.2-68.5 \%$ vs. $42.5-53.6 \%$ ), while adverse event rate was comparable (overall adverse event rate: $94.4 \%$ vs. $95.4 \%$; grade $1-2$ adverse event rate: $35.9 \%$ vs. 45.4\%; grade 3-4 adverse event rate: $55.7 \%$ vs. $47.7 \%$ ) [94]. Besides, the results of Kaplan-Meier analysis showed that both progression-free survival (PFS) and OS were significantly prolonged in $\mathrm{ABCP}$ group (median PFS of $\mathrm{ABCP}$ vs. BCP: 8.3 vs. 6.8 months; hazard ratio: 0.61, 95\% CI: 0.52 to 0.72 ) (median OS of ABCP vs. BCP: 19.2 vs. 14.7 months; hazard ratio: $0.78,95 \%$ CI: 0.64 to 0.96) [94]. Further analysis showed that ABCP group had an obvious advantage in PFS over BCP group 
Table 1 Clinical trials investigating the efficacy of $\mathrm{ICl}$ plus anti-angiogenesis therapy

\begin{tabular}{|c|c|c|c|c|}
\hline Trials Identifier & Disease & Treatment (arm of combination therapy) & Phase & Status \\
\hline NCT03024437 & RCC & Atezolizumab + bevacizumab + entinostat & $|/| \mid$ & Recruiting \\
\hline NCT03363867 & OC & Atezolizumab + bevacizumab + cobimetinib & $\|$ & Recruiting \\
\hline NCT03472560 & NSCLC/UC & Avelumab + axitinib & $\|$ & Recruiting \\
\hline NCT03395899 & BC & Atezolizumab + bevacizumab + cobimetinib & $\|$ & Recruiting \\
\hline NCT02724878 & NCCKC & Atezolizumab + bevacizumab & $\|$ & Recruiting \\
\hline NCT03386929 & NSCLC & Avelumab + axitinib + palbociclib & $|/| \mid$ & Recruiting \\
\hline NCT03574779 & OC & TSR-042+ bevacizumab + Niraparib & $\|$ & Recruiting \\
\hline NCT02921269 & CC & Atezolizumab + bevacizumab & $\|$ & Active, not recruiting \\
\hline NCT03647956 & NSCLC & Atezolizumab + bevacizumab + carboplatin + pemetrexed & $\|$ & Recruiting \\
\hline NCT02734004 & $\mathrm{OC} / \mathrm{BC} / \mathrm{SCLC} / \mathrm{GC}$ & MEDI4736 + bevacizumab + olaparib & $|/| \mid$ & Recruiting \\
\hline NCT03517449 & EC & Pembrolizumab + lenvatinib & III & Recruiting \\
\hline NCT02572687 & GC/GEJ/NSCLC/HCC & MEDI4736 + ramucirumab & । & Active, not recruiting \\
\hline NCT02839707 & OC/FTC/PC & Atezolizumab + bevacizumab + PLD & $\|/\|$ & Recruiting \\
\hline NCT03289533 & HCC & Avelumab + axitinib & । & Recruiting \\
\hline NCT02210117 & RCC & Ipilimumab + bevacizumab & । & Active, not recruiting \\
\hline NCT01950390 & Melanoma & Ipilimumab + bevacizumab & $\|$ & Active, not recruiting \\
\hline NCT03394287 & BC & SHR-1210 + apatinib & $\|$ & Recruiting \\
\hline NCT03417895 & SCLC & SHR-1210 + apatinib & $\|$ & Not yet recruiting \\
\hline NCT03491631 & Multiple solid tumors & SHR-1210 + apatinib + SHR9146 & । & Not yet recruiting \\
\hline NCT02942329 & $\mathrm{HCC} / \mathrm{GC}$ & SHR-1210 + apatinib & |//1 & Recruiting \\
\hline NCT03671265 & ESCC & SHR-1210 + apatinib + radiation & NA & Not yet recruiting \\
\hline NCT03359018 & Osteosarcoma & SHR-1210 + apatinib & $\|$ & Active, not recruiting \\
\hline NCT03722875 & $\mathrm{HCC}$ & SHR-1210 + apatinib & NA & Not yet recruiting \\
\hline NCT03502746 & Mesothelioma & Nivolumab + ramucirumab & $\|$ & Recruiting \\
\hline NCT03606174 & UC & Nivolumab + sitravatinib & $\|$ & Recruiting \\
\hline NCT02853331 & $\mathrm{RCC}$ & Pembrolizumab + axitinib & III & Active, not recruiting \\
\hline NCT03680521 & $\mathrm{RCC}$ & Nivolumab + sitravatinib & $\|$ & Recruiting \\
\hline NCT02493751 & $\mathrm{RCC}$ & Avelumab + axitinib & । & Active, not recruiting \\
\hline NCT02684006 & $\mathrm{RCC}$ & Avelumab + axitinib & III & Active, not recruiting \\
\hline NCT02366143 & NSCLC & Atezolizumab + bevacizumab + paclitaxel + carboplatin & III & Active, not recruiting \\
\hline NCT00790010 & Melanoma & Ipilimumab + bevacizumab & । & Active, not recruiting \\
\hline NCT 01633970 & Multiple solid tumors & Atezolizumab + bevacizumab & । & Active, not recruiting \\
\hline
\end{tabular}

The details of Table 1 was obtained from http://clinicaltrials.gov/. Abbreviations: $B C$ breast cancer, $C C$ cervical cancer, $E C$ endometrial cancer, ESCC esophageal squamous cell carcinoma, FTC fallopian tube cancer, GC gastric cancer, GEJ gastroesophageal junction adenocarcinoma, GIST gastrointestinal stromal tumor, HCC hepatocellular carcinoma, NA not applicable, NCCKC Non-clear cell kidney cancer, NSCLC non-small cell lung cancer, OC ovarian cancer, PC peritoneal cancer, PLD pegylated liposomal doxorubicin hydrochloride, $R C C$ renal cell cancer, SCLC small cell lung cancer, UC urothelial cancer

regardless of PD-L1 expression and effector T cell status [94]. Given that first line atezolizumab treatment is limited to non-small-cell lung cancer patients with high PD-L1 expression, the results of IMpower150 are meaningful to expand the application of ICI [95].

\section{Anti-PD-L1 combined with anti-angiogenesis TKI}

In most clinical studies by far, combination strategies consist of ICI and anti-angiogenesis mAb bevacizumab. In 2018 Choueiri et al. firstly reported the efficacy of avelumab plus anti-angiogenesis TKI axitinib therapy in treatmentnaïve advanced clear-cell renal-cell carcinoma (JAVELIN Renal 100). JAVELIN Renal 100 (NCT02493751) is a phase 1b study aiming to evaluate safety, pharmacokinetics, and pharmacodynamics of avelumab (anti-PD-L1) plus axitinib (VEGFR TKI) therapy [96]. For a total of 55 patients enrolled in the study, 54 patients received avelumab plus axitinib therapy except for one patient due to abnormally increased blood creatine phosphokinase [96]. Within a follow-up period of nearly one year, 58\% (32 of 55) patients 
showed complete response or partial response to combination therapy while $20 \%$ (11 of 55 ) patients had stable disease [96]. Notably, it was observed that PD-L1 expression did not significantly affect treatment efficacy. Whether choosing cut-off value as $1 \%$ or $5 \%$, ORRs of PD-L1 high expression group and PD-L1 low expression group are comparable (cutoff value as $1 \%$ : OR 3.80 , 95\%CI $0.70-$ 18.12; cutoff value as $5 \%$ : OR $2.11,95 \%$ CI $0.60-7.57$ ) [96]. Motivated by the encouraging and preliminary results of NCT02493751, a phase 3 clinical trial JAVELIN Renal 101 (NCT02684006) is ongoing to compare the efficacy of avelumab plus axitinib vs. sunitinib monotherapy in advanced clear-cell renal-cell carcinoma.

Later, $\mathrm{Xu}$ et al. reported the results of another phase 1 clinical study (NCT02942329) which aimed to investigate the efficacy of SHR-1210 (anti-PD-1 antibody) plus apatinib (VEGFR2 TKI) in refractory hepatocellular cancer $(\mathrm{HCC})$, gastric cancer (GC), and esophagogastric junction cancer (EGJC) patients [97]. 15 patients were assigned to dose escalation group and 28 patients were assigned to dose expansion group (recommended phase II dose of apatinib: $250 \mathrm{mg} / \mathrm{d}$ ) [97]. Though the efficacy of combination therapy in GC/EGJC patients was unsatisfactory (ORR in evaluable GC/EGJC: $17.4 \%$ ), the treatment effect in HCC patients was encouraging (ORR in evaluable HCC patients: 50\%, 95\%CI 24.7-75.4\%; disease control rate in evaluable HCC patients: 93.8\%, 95\%CI 69.8-99.8\%; 6-month PFS rate: 51.3\%, 95\%CI 21.4-74.9\%; 9-month PFS rate: $41.0 \%, 95 \%$ CI 13.8 to $66.9 \%$ ) [97]. Compared with the previous data of nivolumab or VEGFR2 TKI monotherapy, patients gained more benefits from combination therapy $[98,99]$. It was presumed that the difference in efficacy among three types of cancers could be attributed to tumor immunogenicity [97]. HCC tends to possess higher immunogenicity than GC and EGJC [97].

\section{Combination therapy-related adverse event}

For ICI therapy, an important factor contributes to treatment discontinuation is the severe adverse event. Most adverse events are related with hyperactive immune response, showing T cell mediated auto-immune like inflammation [100]. Disturbed immune homeostasis results in immune-related damage in normal tissues such as gastrointestinal, skin, and hepatic system [100]. Generally, the risk of anti-PD-1/PD-L1 mAb induced adverse event is lower than anti-CTLA-4 mAb (grade 3-4 adverse event: $7-12 \%$ vs. $10-18 \%$ ) [100]. These adverse events could be alleviated by discontinuing ICI treatment or reducing dose of ICI [64]. Theoretically, anti-angiogenesis promotes tumor vessel normalization, which is favorable to $\mathrm{T}$ cell infiltration and drug delivery to tumor. In the combination therapy, we speculated that lower dose of ICI would be sufficient to counteract immunosuppressive microenvironment with less adverse event [64].

\section{Conclusion}

A series of preclinical and clinical studies indicated the mutually enhanced effect of anti-angiogenesis and ICI therapy. On the one hand, anti-angiogenesis blocks the negative immune signals by increasing ratio of anti-/pro-tumor immune cell and decreasing multiple immune checkpoints expression. On the other hand, ICI therapy could restore immune-supportive microenvironment and promote vessel normalization. Besides, because of enhanced drug delivery benefiting from vessel normalization, smaller dose of ICI could be applied which reduces the risk of adverse event. A main problem needing to resolve is how to optimize the dose and schedule of anti-angiogenesis in the combination therapy. Extending window of vessel normalization and avoiding excessive vessel pruning would facilitate the maximized survival benefit. We believe ICI plus anti-angiogenesis would be a promising strategy to overcome treatment resistance and improve patients' prognosis.

\section{Abbreviations \\ ANGPT-2: Angiopoietin-2; bFGF: Basic fibroblast growth factor; CAR: Chimeric antigen receptor; C-FLIP: Cellular FLICE-inhibitory protein; CTLA-4: Cytotoxic T- lymphocyte antigen-4;, DC: Dendritic cell; EC: Endothelial cell; EGJC: Esophagogastric junction cancer; FasL: Fas ligand; Gal-1: Galectin-1; GC: Gastric cancer; HCC: Hepatocellular cancer; HEV: High endothelial venule; IDO: Indoleamine 2, 3-dioxygenase; IFN-ү: Interferon- $\gamma ;$ IL-10: Interleukin-10; IL-6: Interleukin-6; mAb: Monoclonal antibody; MDSC: Myeloid-derived suppressor cell; MHC-I: Major histocompatibility complex-l; MMP: Matrix metalloproteinase; ORR: Objective response rate; OS: Overall survival; PD- 1: Programmed cell death protein 1; PFS: Progression-free survival; SCLC: Small-cell lung cancer; TAM: Tumor-associated macrophage; TCR: T cell receptor; TIL: Tumor infiltrating lymphocyte; TKI: Tyrosine kinase inhibitor; Treg: Regulatory T cell; VCAM-1: Vasculature cell adhesion molecule-1; VEGF: Vascular endothelial growth factor; VWF: Von Willebrand factor}

\section{Acknowledgements}

Not applicable.

\section{Funding}

This work was supported by the National Natural Science Foundation of China (No. 81874120, 81572608, 81672984), Wuhan Science and Technology Bureau (No. 2017060201010170).

\section{Availability of data and materials}

Data sharing not applicable to this article as no datasets were generated or analyzed during the current study.

\section{Authors' contributions \\ MY performed the selection of literature, drafted the manuscript, and prepared the Figs. DJ, SQ and QC collected the related references and participated in discussion. KW and AL designed this review and revised the manuscript. All authors contributed to this manuscript. All authors read and approved the final manuscript.}

Ethics approval and consent to participate Not applicable.

Consent for publication

Not applicable.

Competing interests

The authors declare that they have no competing interests. 


\section{Publisher's Note}

Springer Nature remains neutral with regard to jurisdictional claims in published maps and institutional affiliations.

Received: 17 December 2018 Accepted: 22 February 2019 Published online: 30 March 2019

\section{References}

1. Topalian SL, Drake CG, Pardoll DM. Targeting the PD-1/B7-H1(PD-L1) pathway to activate anti-tumor immunity. Curr Opin Immunol. 2012;24:207-12.

2. Lipson EJ, Drake CG. Ipilimumab: an anti-CTLA-4 antibody for metastatic melanoma. Clin Cancer Res. 2011;17:6958-62.

3. Long J, Lin J, Wang A, Wu L, Zheng Y, Yang X, et al. PD-1/PD-L blockade in gastrointestinal cancers: lessons learned and the road toward precision immunotherapy. J Hematol Oncol. 2017:10:146.

4. Wang J, Yuan R, Song W, Sun J, Liu D, Li Z. PD-1, PD-L1 (B7-H1) and tumorsite immune modulation therapy: the historical perspective. J Hematol Oncol. 2017:10:34

5. Kythreotou A, Siddique A, Mauri FA, Bower M, Pinato DJ. PD-L1. J Clin Pathol. 2018;71:189-94

6. Yi M, Jiao D, Xu H, Liu Q, Zhao W, Han X, et al. Biomarkers for predicting efficacy of PD-1/PD-L1 inhibitors. Mol Cancer. 2018;17:129.

7. Patsoukis N, Brown J, Petkova V, Liu F, Li L, Boussiotis VA. Selective effects of PD-1 on Akt and Ras pathways regulate molecular components of the cell cycle and inhibit T cell proliferation. Sci Signal. 2012;5:ra46.

8. Yi M, Qin S, Zhao W, Yu S, Chu Q, Wu K. The role of neoantigen in immune checkpoint blockade therapy. Exp Hematol Oncol. 2018;7:28.

9. Li X, Shao C, Shi Y, Han W. Lessons learned from the blockade of immune checkpoints in cancer immunotherapy. J Hematol Oncol. 2018;11:31.

10. Marin-Acevedo JA, Soyano AE, Dholaria B, Knutson KL, Lou Y. Cancer immunotherapy beyond immune checkpoint inhibitors. J Hematol Oncol. 2018;11:8.

11. Furness AJ, Vargas FA, Peggs KS, Quezada SA. Impact of tumour microenvironment and $\mathrm{fc}$ receptors on the activity of immunomodulatory antibodies. Trends Immunol. 2014;35:290-8.

12. Arce Vargas F, Furness AJS, Litchfield K, Joshi K, Rosenthal R, Ghorani E, et al. Fc Effector Function Contributes to the Activity of Human Anti-CTLA-4 Antibodies. Cancer Cell. 2018;33:649-63.e4.

13. Romano E, Kusio-Kobialka M, Foukas PG, Baumgaertner P, Meyer C, Ballabeni $P$, et al. Ipilimumab-dependent cell-mediated cytotoxicity of regulatory $T$ cells ex vivo by nonclassical monocytes in melanoma patients. Proc Natl Acad Sci U S A. 2015:112:6140-5.

14. Ramjiawan RR, Griffioen AW, Duda DG. Anti-angiogenesis for cancer revisited: is there a role for combinations with immunotherapy? Angiogenesis. 2017;20:185-204

15. Kerbel RS. Tumor angiogenesis. N Engl J Med. 2008:358:2039-49.

16. Sun W. Angiogenesis in metastatic colorectal cancer and the benefits of targeted therapy. J Hematol Oncol. 2012:5:63.

17. Ronca R, Benkheil M, Mitola S, Struyf S, Liekens S. Tumor angiogenesis revisited: regulators and clinical implications. Med Res Rev. 2017:37:1231-74.

18. Sato Y. Molecular diagnosis of tumor angiogenesis and anti-angiogenic cancer therapy. Int J Clin Oncol. 2003;8:200-6.

19. Jain RK. Molecular regulation of vessel maturation. Nat Med. 2003;9:685-93.

20. Dvorak HF. Vascular permeability factor/vascular endothelial growth factor: a critical cytokine in tumor angiogenesis and a potential target for diagnosis and therapy. J Clin Oncol. 2002;20:4368-80.

21. Falcon BL, O'Clair B, McClure D, Evans GF, Stewart J, Swearingen ML, et al. Development and characterization of a high-throughput in vitro cord formation model insensitive to VEGF inhibition. J Hematol Oncol. 2013:6:31.

22. Cooke VG, LeBleu VS, Keskin D, Khan Z, O'Connell JT, Teng Y, et al. Pericyte depletion results in hypoxia-associated epithelial-to-mesenchymal transition and metastasis mediated by met signaling pathway. Cancer Cell. 2012;21:66-81.

23. Ye W. The complexity of translating anti-angiogenesis therapy from basic science to the clinic. Dev Cell. 2016:37:114-25.

24. Hegde PS, Karanikas V, Evers S. The where, the when, and the how of immune monitoring for Cancer immunotherapies in the era of checkpoint inhibition. Clin Cancer Res. 2016;22:1865-74.

25. Yagi T, Baba Y, Ishimoto T, Iwatsuki M, Miyamoto Y, Yoshida N, et al. PD-L1 expression, tumor-infiltrating lymphocytes, and clinical outcome in patients with surgically resected esophageal Cancer. Ann Surg. 2017. https://doi.org/ 10.1097/SLA.0000000000002616.
26. Wallin JJ, Bendell JC, Funke R, Sznol M, Korski K, Jones S, et al. Atezolizumab in combination with bevacizumab enhances antigen-specific T-cell migration in metastatic renal cell carcinoma. Nat Commun. 2016;7:12624.

27. Gajewski TF, Woo SR, Zha Y, Spaapen R, Zheng Y, Corrales L, et al. Cancer immunotherapy strategies based on overcoming barriers within the tumor microenvironment. Curr Opin Immunol. 2013;25:268-76.

28. Chen DS, Mellman I. Oncology meets immunology: the cancer-immunity cycle. Immunity. 2013;39:1-10.

29. Somasundaram A, Burns TF. The next generation of immunotherapy: keeping lung cancer in check. J Hematol Oncol. 2017;10:87.

30. Melero I, Rouzaut A, Motz GT, Coukos G. T-cell and NK-cell infiltration into solid tumors: a key limiting factor for efficacious cancer immunotherapy. Cancer Discov. 2014:4:522-6.

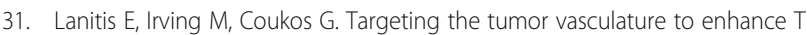
cell activity. Curr Opin Immunol. 2015;33:55-63.

32. Teng MW, Ngiow SF, Ribas A, Smyth MJ. Classifying cancers based on T-cell infiltration and PD-L1. Cancer Res. 2015:75:2139-45.

33. Liu M, Wang X, Wang L, Ma X, Gong Z, Zhang S, et al. Targeting the IDO1 pathway in cancer: from bench to bedside. J Hematol Oncol. 2018;11:100,

34. Gabrilovich DI, Chen HL, Girgis KR, Cunningham HT, Meny GM, Nadaf S, et al. Production of vascular endothelial growth factor by human tumors inhibits the functional maturation of dendritic cells. Nat Med. 1996;2:1096-103.

35. Huang Y, Chen X, Dikov MM, Novitskiy SV, Mosse CA, Yang L, et al. Distinct roles of VEGFR-1 and VEGFR-2 in the aberrant hematopoiesis associated with elevated levels of VEGF. Blood. 2007;110:624-31.

36. Curiel TJ, Coukos G, Zou L, Alvarez X, Cheng P, Mottram P, et al. Specific recruitment of regulatory $T$ cells in ovarian carcinoma fosters immune privilege and predicts reduced survival. Nat Med. 2004;10:942-9.

37. Facciabene A, Peng X, Hagemann IS, Balint K, Barchetti A, Wang LP, et al. Tumour hypoxia promotes tolerance and angiogenesis via CCL28 and T (reg) cells. Nature. 2011;475:226-30

38. Movahedi K, Laoui D, Gysemans C, Baeten M, Stange G, Van den Bossche J, et al. Different tumor microenvironments contain functionally distinct subsets of macrophages derived from Ly6C(high) monocytes. Cancer Res. 2010;70:5728-39.

39. Motz GT, Santoro SP, Wang LP, Garrabrant T, Lastra RR, Hagemann IS, et al. Tumor endothelium FasL establishes a selective immune barrier promoting tolerance in tumors. Nat Med. 2014;20:607-15.

40. Shibuya M. VEGFR and type-V RTK activation and signaling. Cold Spring Harb Perspect Biol. 2013;5:a009092.

41. Basagiannis D, Zografou S, Murphy C, Fotsis T, Morbidelli L, Ziche M, et al. VEGF induces signalling and angiogenesis by directing VEGFR2 internalisation through macropinocytosis. J Cell Sci. 2016;129:4091-104.

42. Abhinand CS, Raju R, Soumya SJ, Arya PS, Sudhakaran PR. VEGF-ANEGFR2 signaling network in endothelial cells relevant to angiogenesis. J Cell Commun Signal. 2016;10:347-54

43. Kendrew J, Eberlein C, Hedberg B, McDaid K, Smith NR, Weir HM, et al. An antibody targeted to VEGFR-2 Ig domains 4-7 inhibits VEGFR-2 activation and VEGFR-2-dependent angiogenesis without affecting ligand binding. Mol Cancer Ther. 2011;10:770-83.

44. Claesson-Welsh L, Welsh M. VEGFA and tumour angiogenesis. J Intern Med. 2013;273:114-27.

45. Keating GM. Bevacizumab: a review of its use in advanced cancer. Drugs. 2014;74:1891-925

46. Singh SR, Dogra A, Stewart M, Das T, Chhablani J. Intravitreal Ziv-Aflibercept: clinical effects and economic impact. Asia Pac J Ophthalmol (Phila). 2017;6:561-8.

47. Tada Y, Togashi Y, Kotani D, Kuwata T, Sato E, Kawazoe A, et al. Targeting VEGFR2 with Ramucirumab strongly impacts effector/ activated regulatory $T$ cells and CD8(+) T cells in the tumor microenvironment. J Immunother Cancer. 2018;6:106.

48. Keating GM. Axitinib: a review in advanced renal cell carcinoma. Drugs. 2015:75:1903-13.

49. Motzer RJ, Escudier B, Gannon A, Figlin RA. Sunitinib: ten years of successful clinical use and study in advanced renal cell carcinoma. Oncologist. 2017:22:41-52.

50. Gravina GL, Mancini A, Marampon F, Colapietro A, Delle Monache S, Sferra $\mathrm{R}$, et al. The brain-penetrating CXCR4 antagonist, PRX177561, increases the antitumor effects of bevacizumab and sunitinib in preclinical models of human glioblastoma. J Hematol Oncol. 2017;10:5.

51. Scott EN, Meinhardt G, Jacques C, Laurent D, Thomas AL. Vatalanib: the clinical development of a tyrosine kinase inhibitor of angiogenesis in solid tumours. Expert Opin Investig Drugs. 2007;16:367-79. 
52. Chinnasamy D, Yu Z, Kerkar SP, Zhang L, Morgan RA, Restifo NP, et al. Local delivery of interleukin-12 using T cells targeting VEGF receptor-2 eradicates multiple vascularized tumors in mice. Clin Cancer Res. 2012;18:1672-83.

53. Jain RK. Normalization of tumor vasculature: an emerging concept in antiangiogenic therapy. Science. 2005;307:58-62.

54. Viallard C, Larrivee B. Tumor angiogenesis and vascular normalization: alternative therapeutic targets. Angiogenesis. 2017;20:409-26.

55. Benjamin LE, Golijanin D, Itin A, Pode D, Keshet E. Selective ablation of immature blood vessels in established human tumors follows vascular endothelial growth factor withdrawal. J Clin Invest. 1999;103:159-65.

56. Ebos JM, Lee CR, Cruz-Munoz W, Bjarnason GA, Christensen JG, Kerbel RS. Accelerated metastasis after short-term treatment with a potent inhibitor of tumor angiogenesis. Cancer Cell. 2009;15:232-9.

57. Paez-Ribes M, Allen E, Hudock J, Takeda T, Okuyama H, Vinals F, et al. Antiangiogenic therapy elicits malignant progression of tumors to increased local invasion and distant metastasis. Cancer Cell. 2009;15:220-31.

58. Hurwitz H, Fehrenbacher L, Novotny W, Cartwright T, Hainsworth J, Heim W, et al. Bevacizumab plus irinotecan, fluorouracil, and leucovorin for metastatic colorectal cancer. N Engl J Med. 2004;350:2335-42.

59. Huang Y, Goel S, Duda DG, Fukumura D, Jain RK. Vascular normalization as an emerging strategy to enhance cancer immunotherapy. Cancer Res. 2013, 73:2943-8.

60. Kleibeuker EA, Ten Hooven MA, Verheul HM, Slotman BJ, Thijssen VL. Combining radiotherapy with sunitinib: lessons (to be) learned. Angiogenesis. 2015;18:385-95.

61. Folkman J. Tumor angiogenesis: therapeutic implications. N Engl J Med. 1971;285:1182-6.

62. Huang Y, Yuan J, Righi E, Kamoun WS, Ancukiewicz M, Nezivar J, et al. Vascular normalizing doses of antiangiogenic treatment reprogram the immunosuppressive tumor microenvironment and enhance immunotherapy. Proc Natl Acad Sci U S A. 2012;109:17561-6.

63. Jain RK. Antiangiogenesis strategies revisited: from starving tumors to alleviating hypoxia. Cancer Cell. 2014;26:605-22.

64. Fukumura D, Kloepper J, Amoozgar Z, Duda DG, Jain RK. Enhancing cance immunotherapy using antiangiogenics: opportunities and challenges. Nat Rev Clin Oncol. 2018;15:325-40.

65. Du Four S, Maenhout SK, Niclou SP, Thielemans K, Neyns B, Aerts JL. Combined VEGFR and CTLA-4 blockade increases the antigen-presenting function of intratumoral DCs and reduces the suppressive capacity of intratumoral MDSCs. Am J Cancer Res. 2016:6:2514-31.

66. Horikawa N, Abiko K, Matsumura N, Hamanishi J, Baba T, Yamaguchi K, et al. Expression of vascular endothelial growth factor in ovarian Cancer inhibits tumor immunity through the accumulation of myeloid-derived suppressor cells. Clin Cancer Res. 2017:23:587-99.

67. Noman MZ, Desantis G, Janji B, Hasmim M, Karray S, Dessen P, et al. PD-L1 is a novel direct target of HIF-1alpha, and its blockade under hypoxia enhanced MDSC-mediated T cell activation. J Exp Med. 2014;211:781-90.

68. Kammertoens T, Friese C, Arina A, Idel C, Briesemeister D, Rothe M, et al. Tumour ischaemia by interferon-gamma resembles physiological blood vessel regression. Nature. 2017:545:98-102.

69. Huang Y, Kim BYS, Chan CK, Hahn SM, Weissman IL, Jiang W. Improving immune-vascular crosstalk for cancer immunotherapy. Nat Rev Immunol. 2018;18:195-203.

70. Tian L, Goldstein A, Wang H, Ching Lo H, Sun Kim I, Welte T, et al. Mutual regulation of tumour vessel normalization and immunostimulatory reprogramming. Nature. 2017;544:250-4.

71. Yasuda S, Sho M, Yamato I, Yoshiji H, Wakatsuki K, Nishiwada S, et al. Simultaneous blockade of programmed death 1 and vascular endothelial growth factor receptor 2 (VEGFR2) induces synergistic anti-tumour effect in vivo. Clin Exp Immunol. 2013;172:500-6.

72. Wu FTH, Xu P, Chow A, Man S, Kruger J, Khan KA, et al. Pre- and postoperative anti-PD-L1 plus anti-angiogenic therapies in mouse breast or renal cancer models of micro- or macro-metastatic disease. $\mathrm{Br} J$ Cancer. 2018. https://doi.org/10.1038/s41416-018-0297-1.

73. Meder L, Schuldt P, Thelen M, Schmitt A, Dietlein F, Klein S, et al. Combined VEGF and PD-L1 blockade displays synergistic treatment effects in an autochthonous mouse model of small cell lung Cancer. Cancer Res. 2018;78: 4270-81.

74. Voron T, Colussi O, Marcheteau E, Pernot S, Nizard M, Pointet AL, et al. VEGF-A modulates expression of inhibitory checkpoints on CD8+ T cells in tumors. J Exp Med. 2015;212:139-48.
75. Wu X, Giobbie-Hurder A, Liao X, Connelly C, Connolly EM, Li J, et al. Angiopoietin-2 as a biomarker and target for immune checkpoint therapy. Cancer Immunol Res. 2017;5:17-28.

76. D'Amico G, Korhonen EA, Anisimov A, Zarkada G, Holopainen T, Hagerling $\mathrm{R}$, et al. Tie1 deletion inhibits tumor growth and improves angiopoietin antagonist therapy. J Clin Invest. 2014;124:824-34.

77. Rigamonti N, Kadioglu E, Keklikoglou I, Wyser Rmili C, Leow CC, De Palma M. Role of angiopoietin-2 in adaptive tumor resistance to VEGF signaling blockade. Cell Rep. 2014;8:696-706.

78. Schmittnaegel M, Rigamonti N, Kadioglu E, Cassara A, Wyser Rmili C, Kiialainen A, et al. Dual angiopoietin-2 and VEGFA inhibition elicits antitumor immunity that is enhanced by PD-1 checkpoint blockade. Sci Transl Med. 2017. https://doi.org/10.1126/scitranslmed.aak9670.

79. Allen E, Jabouille A, Rivera LB, Lodewijckx I, Missiaen R, Steri V, et al. Combined antiangiogenic and anti-PD-L1 therapy stimulates tumor immunity through HEV formation. Sci Transl Med. 2017. https://doi.org/10. 1126/scitransImed.aak9679.

80. Ager A, May MJ. Understanding high endothelial venules: lessons for cancer immunology. Oncoimmunology. 2015;4:e1008791.

81. Ager A. High endothelial Venules and other blood vessels: critical regulators of lymphoid organ development and function. Front Immunol. 2017:8:45.

82. Hayasaka H, Taniguchi K, Fukai S, Miyasaka M. Neogenesis and development of the high endothelial venules that mediate lymphocyte trafficking. Cancer Sci. 2010;101:2302-8

83. Johansson-Percival A, He B, Ganss R. Immunomodulation of tumor vessels: it takes two to tango. Trends Immunol. 2018;39:801-14.

84. Schmidt EV. Developing combination strategies using PD-1 checkpoint inhibitors to treat cancer. Semin Immunopathol. 2019;41:21-30.

85. Hodi FS, Lawrence D, Lezcano C, Wu X, Zhou J, Sasada T, et al. Bevacizumab plus ipilimumab in patients with metastatic melanoma. Cancer Immunol Res. 2014;2:632-42.

86. Wu X, Giobbie-Hurder A, Liao X, Lawrence D, McDermott D, Zhou J, et al. VEGF neutralization plus CTLA-4 blockade alters soluble and cellular factors associated with enhancing lymphocyte infiltration and humoral recognition in melanoma. Cancer Immunol Res. 2016;4:858-68.

87. Hodi FS, O'Day SJ, McDermott DF, Weber RW, Sosman JA, Haanen JB, et al. Improved survival with ipilimumab in patients with metastatic melanoma. N Engl J Med. 2010;363:711-23.

88. Wu X, Li J, Connolly EM, Liao X, Ouyang J, Giobbie-Hurder A, et al. Combined anti-VEGF and anti-CTLA-4 therapy elicits humoral immunity to Galectin-1 which is associated with favorable clinical outcomes. Cancer Immunol Res. 2017:5:446-54

89. Astorgues-Xerri L, Riveiro ME, Tijeras-Raballand A, Serova M, Neuzillet C, Albert S, et al. Unraveling galectin-1 as a novel therapeutic target for cancer. Cancer Treat Rev. 2014:40:307-19.

90. Ho WL, Hsu WM, Huang MC, Kadomatsu K, Nakagawara A. Protein glycosylation in cancers and its potential therapeutic applications in neuroblastoma. J Hematol Oncol. 2016;9:100.

91. Croci DO, Cerliani JP, Dalotto-Moreno T, Mendez-Huergo SP, Mascanfroni ID, Dergan-Dylon S, et al. Glycosylation-dependent lectinreceptor interactions preserve angiogenesis in anti-VEGF refractory tumors. Cell. 2014;156:744-58

92. McDermott DF, Sosman JA, Sznol M, Massard C, Gordon MS, Hamid O, et al. Atezolizumab, an anti-programmed death-ligand 1 antibody, in metastatic renal cell carcinoma: Long-term safety, clinical activity, and immune correlates from a phase la study. J Clin Oncol. 2016;34:833-42.

93. Yang JC, Haworth L, Sherry RM, Hwu P, Schwartzentruber DJ, Topalian $\mathrm{SL}$, et al. A randomized trial of bevacizumab, an anti-vascular endothelial growth factor antibody, for metastatic renal cancer. $\mathrm{N}$ Engl J Med. 2003:349:427-34.

94. Socinski MA, Jotte RM, Cappuzzo F, Orlandi F, Stroyakovskiy D, Nogami N, et al. Atezolizumab for first-line treatment of metastatic nonsquamous NSCLC. N Engl J Med. 2018;378:2288-301.

95. Ryu R, Ward KE. Atezolizumab for the first-line treatment of non-small cell lung Cancer (NSCLC): current status and future prospects. Front Oncol. 2018; 8:277.

96. Choueiri TK, Larkin J, Oya M, Thistlethwaite F, Martignoni M, Nathan P, et al. Preliminary results for avelumab plus axitinib as first-line therapy in patients with advanced clear-cell renal-cell carcinoma (JAVELIN renal 100): an openlabel, dose-finding and dose-expansion, phase 1b trial. Lancet Oncol. 2018; 19:451-60. 
97. Xu J, Zhang Y, Jia R, Yue C, Chang L, Liu R, et al. Anti-PD-1 antibody SHR1210 combined with Apatinib for advanced hepatocellular carcinoma, gastric, or Esophagogastric junction Cancer: an open-label, dose escalation and expansion study. Clin Cancer Res. 2019;25:515-23.

98. El-Khoueiry AB, Sangro B, Yau T, Crocenzi TS, Kudo M, Hsu C, et al. Nivolumab in patients with advanced hepatocellular carcinoma (CheckMate 040): an open-label, non-comparative, phase 1/2 dose escalation and expansion trial. Lancet. 2017;389:2492-502.

99. Llovet JM, Ricci S, Mazzaferro V, Hilgard P, Gane E, Blanc JF, et al. Sorafenib in advanced hepatocellular carcinoma. N Engl J Med. 2008;359:378-90.

100. Naidoo J, Page DB, Li BT, Connell LC, Schindler K, Lacouture ME, et al. Toxicities of the anti-PD-1 and anti-PD-L1 immune checkpoint antibodies. Ann Oncol. 2015;26:2375-91.

Ready to submit your research? Choose BMC and benefit from:

- fast, convenient online submission

- thorough peer review by experienced researchers in your field

- rapid publication on acceptance

- support for research data, including large and complex data types

- gold Open Access which fosters wider collaboration and increased citations

- maximum visibility for your research: over $100 \mathrm{M}$ website views per year

At BMC, research is always in progress.

Learn more biomedcentral.com/submissions 